\title{
ON UNIFORM CONNECTEDNESS
}

\section{BABOOLAL}

\author{
Department of Mathematics \\ University of Durban-Westrille \\ Private Bag X54001, Durban 4000 \\ South Africa
}

(Received July 6, 1987 and in revised form August 29, 1988)

\begin{abstract}
The concept of unfform connectedness, which generalizes the concept of well-chainedness for metric spaces, is used to prove the following: (a) If two points $a$ and $b$ of a compact Hausdorff uniform space $(X, U)$ can be joined by a U-chain for every $U \varepsilon U$, then they lie together in the same component of $X$; (b) Let ( $x, U)$ be a compact Hausdorff uniform space, $A$ and $B$ non-empty disjoint closed subsets of $X$ such that no component of $X$ intersects both $A$ and $B$. Then there exists a separation $X=X_{A} \cup X_{B}$, where $X_{A}$ and $X_{B}$ are disjoint compact sets containing $A$ and $B$ respectively. These generalize the corresponding results for metric spaces.
\end{abstract}

KEY WORDS AND PHRASES. Uniform spaces, uniformly connectedness and we11-chainedness. 1980 AMS SUBJECT CLASSIFICATION CODES. Primary 54E15, Secondary 54D05.

\section{INTRODUCTION.}

All topological spaces and all uniform spaces considered are Hausdorff, (X,U) being used to deote a uniform space with $U$ the family of entourages of $X$. In [1] Mrowka and Pervin introduced into the theory of uniform spaces the concept of uniform connectedness: $(X, U)$ is uniformly connected iff every uniformly continuous function on $X$ to a discrete uniform space is constant. As is evident from the definition, connectedness is a stronger property than uniform connectedness. However, for the class of compact uniform spaces these two properties are equivalent [1].

In [2] a criterion for a completely regular Hausdorff space to have a locally connected compactification was obtained. This criterion, which involved the concept of uniform connectedness, is that the space must possess a compatible uniformity with respect to which it has weak property S. A uniform space $(X, U)$ is said to have weak property $S$ if given $U \varepsilon U, X$ can be covered by a finite collection of uniformly connected sets each of which is U-smal1 [2]. In view of the fact that the concept of uniform connectedness bears on the problem of existence of locally connected compactifications a further study of this concept was felt to be justified. Our specific aim in this paper is to show how we can use this concept to generalize to compact Hausdorff uniform spaces the following two propositions appearing in [3], pp. 15. 
PROPOSITION 1.1. If two points $a$ and $b$ of a compact metric space $x$ can be joined by an $\varepsilon$-chain for every $\varepsilon>0$, they lie together in the same component of $X$.

PROPSITION 1.2. If $A$ and $B$ are disjoint closed sets of a compact metric space $X$ such that no component of $X$ intersects both $A$ and $B$, there exists a separation $X=X_{A} \cup X_{B}$ where $X_{A}$ and $X_{B}$ are disjoint compact sets containing $A$ and $B$ respectively. Along the way a problem in [4], p. 170 is strengthened.

\section{PREL IMINARIES.}

We need some basic facts on Efremovi č proximities, (EF-proximities, for short). Proofs of these can be found in [5]. If $(X, \delta)$ is an EF-proximity space, its associated topology defined by

$$
T(\delta)=\{G \subset X \mid \times \phi(X-G) \text { for each } x \in G\}
$$

is completely regular and is Hausdorff iff $(X, \delta)$ is separated. Conversely every completely regular space $(X, T)$ admits an Ef-proximity $\delta$ such that $T(\delta)=T$. Each uniform space $(X, U)$ has an associated EF-proximity $\delta=\delta(U)$ given by $A \delta B \Leftrightarrow(A x B) \cap U / \neq 0$ for every $U \varepsilon U$. Furthermore the uniform topology $T(W=T(\delta)$.

As pointed out in [1], (topologica1) connectedness implies uniform connectedness but the convese is not true in general, the rational numbers with the usual uniformity providing a counterexample.

We now define the concept of a well-chained uniform space which Mrowka and Pervin have called 'chain-connected' in [1]. This is a natural generalization of the concept of we11-chained metric spaces (see eg [3]).

DEFINITION 2.1. A U-chain from a point $x \in X$ to a point $y \varepsilon X$ in a uniform space $(X, U)$, where $U \varepsilon U$, is a finite sequence of points $x=x_{1}, x_{2}, \ldots, x_{n}=y$ such that

$\left(x_{1}, x_{1+1}\right) \varepsilon U$ for each $1=1,2, \ldots, n-1 .(x, U)$ is said to be well-chained iff any two points $x$ and $y$ in $X$ can be joined by a U-chain for every $U \in U$.

We require the following two results due to Mowka and Pervin [1].

THEOREM 2.2. Let $(X, U)$ be a uniform space and $\delta$ the induced proximity relation. The following conditions are equivalent:

(a) $(X, U)$ is uniformly connected

(b) For every $A \subset X, \phi \neq A \neq X$, we have $A \delta(X-A)$

(c) $(X, U)$ is well-chained.

THEOREM 2.3. A uniform space $(X, U)$ is connected iff it is uniformly connected with respect to every uniformity compatible with its topology. Thus a compact uniform space which is uniformly connected mist be connected, as it has a unique compatible uniformity.

In [6], Sieber and Pervin discuss connectedness in syntopogeneous spaces (which generalize the notions of topological, proximity and uniform spaces; see [5] eg.). The following results which we have stated in the context of uniform spaces, have been obtained in the general setting of syntopogenous spaces in [6], and are stated here for subsequent reference. First a definition. 
DEFINITION 2.4. Two sets $A$ and $B$ in a uniform spaces $(X, U)$ are said to be uniformly separated iff $A B$, where $\delta=\delta(U)$ is the induced EF-proximity.

THEOREM $2.5[6]$. (a) The uniformly continuous image of a uniformly connected space is again uniformly connected.

(b) If $A$ is a uniformly connected subset of $(X, U)$ and $A \subset(M \cup N)$ where $M$ and $N$ are uniformly separated subsets of $X$, then either $A \subset M$ or $A \subset N$.

(c) If $A$ is a uniformly connected subset of $(X, U)$ and $B \subset X$ is such that $A \subset B$ $C \bar{A}$, then $B$ is uniformly connected.

(d) If $\left\{A_{\lambda} \mid \lambda \varepsilon \Lambda\right)$ is a family of uniformly connected subsets of (X,U) and $A_{\lambda_{0}}\left(\lambda_{0} \varepsilon \Lambda\right)$ is not uniformly separated from any $A_{\lambda},\left(\lambda \neq \lambda_{0}\right)$, then $A=\bigcup_{\lambda}^{U} A_{\lambda}$ is uniformly connected.

From (d) above it follows that the union of all uniform connected subsets of $X$ which contain a fixed point $p \in X$ is a uniformly connected set, which is called the unfform component of $p$ denoted by $c^{U}(p)$. It is clearly the maximal uniformly connected set containing p.

If $c^{U}(p)$ and $c^{U}(q)$ are not uniformly separated, then by (d), $c^{U}(p) \cup c^{U}$ (q) is uniformly connected, and by maximality we have $c^{U}(p)=c^{U}(q)$. Thus a uniform space is partitioned into uniformly separated uniform components. Clearly by (c) above these uniform components are closed sets. Since each connected set is uniformly connected, the component $C_{p}$ containing peX is clearly contained in $C^{U}(p)$. An example where $c^{U}(\mathrm{p})$ is strictly larger than $C_{p}$ is easily found: Let $Q$ be the rational numbers with the usual uniformity. Then $C_{p}=\{p\}$ for each $p \in Q$, but $C U$ (p) $=$ $Q$ since $Q$ is uniformly connected as was remarked in [1].

3. THE RESULTS.

Let $(X, U)$ be a uniform space. For each subset $A$ of $X$ and $U \varepsilon U$ let $C_{U}(A)=\{x \in X \mid x$ can be joined to some point of $A$ by $a$-chain $\}$ and let $C(A)=\bigcap_{U \in U} C_{U}(A)$

We thus have the following result which is a generalization of problem $T(a)$ in [4] $p$. 170.

PROPOSITION 3.1. For each $A \subset X, U \in U, C_{U}(A)$ is clopen.

PROOF. To show $C_{U}(A)$ open, let $x \in C_{U}(A)$. Find a $\varepsilon A$ and a U-chain $x=x_{1}, x_{2}, \ldots, x_{n}=$ a joining $x$ to a. Find open symmetric $v \varepsilon U$ such that $V \subset U$. Then $x \in V[x] \subset C_{U}(A)$ : for if $y \in V[x]$ then $(x, y) \varepsilon V$ and hence $(y, x) \varepsilon V \subset U$. Thus $y, x_{1}, x_{2}, \ldots, x_{n}=a$ is a U-chain joining $y$ to a and hence $y \varepsilon C_{U}(A)$, thus showing $C_{U}(A)$ open.

To show $C_{U}(A)$ closed, let $y \in \overline{C_{U}(A)}$. Find $x \in C_{U}$ (A) such that $x \in U[y]$. There exists a U-chain $x=x_{1}, x_{2}, \ldots, x_{n}=a$ joining $x$ to some point $a \varepsilon A$, and hence $y, x_{1}, x_{2}, \ldots, x_{n}=$ a is a U-chain joining y to a $\varepsilon A$, ie y $\varepsilon C_{U}(A)$, thus showing $C_{U}(A)$ is closed. 
The next proposition proved in the setting of uniform spaces, when interpreted in the setting of metric spaces, strengthens Problem $T(b)$ of [4], p. $170 \mathrm{v} 1 \mathrm{z}$, that for a compact metric space $X$, if $A C X$ is connected, then $C(A)$ is connected, $C$ ( $A$ ) being defined as $\bigcap_{\varepsilon}{ }_{0} C_{\varepsilon}(A)$ for metric spaces. It is of course a strengthening since $A$ may be take to be uniformly connected instead of (the stronger) connected in the hypothesis. Use is made of this result in the generalizations of Proposition 1.1 and 1.2. First we need the following lemma which can be easily proved or can be found in [4], p. 163, Problem F(a).

LEMMA 3.2. Let $F$ be a family of closed compact sets in a topological space such that $\cap\{A \mid A \varepsilon F\} \subset U$ where $U$ is open. Then there exists a finite subfamily $\mathcal{H}$ of $\mathcal{f}$ such that $\bigcap\{A \mid A E H\} \subset U$.

PROPOSITION 3.3. Let $(X, U)$ be a compact uniform space, $A \subset X$ uniformly connected. Then $C(A)$ is connected.

PROOF. Suppose $C(A)$ is disconnected. Now since $C(A)=\cap_{U \in \mathcal{U} U} C_{U}(A)$ and $C_{U}(A)$ is closed for each UEU by Proposition 3.1, C(A) is closed in $X$ and hence compact. This implies by Theorem 2.3 that $C(A)$ is not uniformly connected. By Theorem $2.2 C(A)=$ $B \cup D$ where $B \neq \phi, D \neq \phi$ and $B \not D$, where $\delta=\delta(U)$ is the EF-proximity induced by $U$.

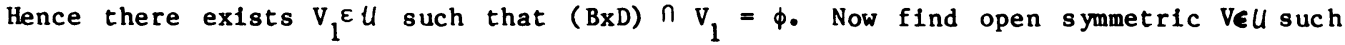
that $V \circ V \circ V \subset V_{1}$. Noting that each $C_{U}(A)$ is closed and compact and that $C(A)$ $=\stackrel{n}{U \varepsilon U C_{U}(A) \subset V[B] U V[D]}$ we have, by the above lemma, a finite collection $\left\{G_{U_{1}}(A)\right\}_{i=1}^{n}, U_{i} \in U$, such that

$$
\bigcap_{i=1}^{n} C_{U_{1}}(A) \subset V[B] U V[D]
$$

Let $U=\bigcap_{i=1}^{n} U_{i}$. Then $U \in U$ and clearly we have

$$
C_{U}(A) \bigcap_{i=1}^{n} C_{U_{i}}(A) \subset V[B] \cup V[D] .
$$

We may assime without loss of generality that $U \subset V$. Now since $A$ is uniformly connected and $A \subset C(A)=B \cup D$ with $B \not D$ we have from Theorem $2.5(b)$ that either $A \subset B$ or $A \subset D$. Lets assume $A \subset D$.

Since $B=\phi$, find $x \in B$. Then $x \in C(A) \subset C_{U}(A)$ and hence there exists a $U$-chain $x=x_{1}, x_{2}, \ldots, x_{n}=a$ joining $x$ to some point a $\varepsilon A$. Clearly each $x_{1}$, for $1<1<n$, belongs to $C_{U}(A)$, and hence $\left\{x_{1}, x_{2}, \ldots, x_{n}\right\} \subset V[B] \cup V[D]$. As is easily verified $V[B] \cap V[D]=\phi$.

Now let $x_{k}$ be the last point in this chain such that $x_{k} \varepsilon V[B]$. Then $x_{k} \neq x_{n}=a$ otherwise $V[B] \cap V[D] \neq \phi$. Thus $x_{k+1}$ belongs to this chain and $x_{k+1} \varepsilon V[D]$. Thus we can find $b \varepsilon B, d \in D$ such that $x_{k} \varepsilon V[b]$ and $x_{k+1} \varepsilon V[d]$. Since $\left(x_{k}, x_{k+1}\right) \varepsilon U \subset V$, we have $(b, d) \varepsilon V \circ V \circ V \subset V_{1}$ contradicting the fact that $(B \times D) \cap v_{1}=\phi$. Hence $C(A)$ is connected. 
Noting that every connected set is well chained we have:

COROLlaRY 3.4. If $(X, U)$ is a compact uniform space and $x \in X$, then $C(\{x\})=C_{x}$, the component of $x$ in $X$.

We now genralize Proposition 1.1 .

COROLLARY 3.5. If two points $a$ and $b$ of a compact uniform space $(X, U)$ can be joined by a U-chain for every $U \varepsilon U$, then they lie together in the same component of $X$.

PROOF. From Corollary 3.4, $C(\{a\})=C_{a}, C(\{b\})=C_{b}$. By hypothesis a $\varepsilon C(\{b\})$. Thus $\mathrm{C}_{a} \cap \mathrm{C}_{b} \neq \phi$ and hence $\mathrm{C}_{\mathrm{a}}=\mathrm{C}_{\mathrm{b}}$.

We remark that the condition that $X$ is compact cannot be dropped, the rationals with the usual uniformity providing an easy counterexample.

The next result generalizes Proposition 1.2 .

THEOREM 3.6. Let $(X, U)$ be a compact uniform space, A and B non-empty disjoint closed subsets of $X$ such that no component of $X$ intersects both $A$ and $B$. Then there exists a separation $X=X_{A} \cup X_{B}$, where $X_{A}$ and $X_{B}$ are disjoint compact sets containing $A$ and $B$ respectively.

PROOF. We claim there exists $U \in U$ such that no $U$-chain in $X$ joins a point of $A$ to a point of B. For suppose not. Then for each $U \varepsilon U$, there exists $a_{U} \varepsilon A$ and $b_{U} \varepsilon B$ such that there is a U-chain in $X$ joining $a_{U}$ to $b_{U}$. Now $(U,<)$ is a directed set according to the definition $U_{1}<U_{2}$ iff $U_{1} \supset U_{2}$. Consequently ( $a_{U}$ ) and (b ${ }_{U}$ ) are nets in $A$ and $B$ respectively. Now $\left(a_{U}\right)$ has a cluster point a $A$ since $A$ is compact. Let $\left(a_{U_{\lambda}}\right)$ be a subner of $\left(a_{U}\right)$ such $a_{U_{\lambda}}+a_{\text {. Then }}\left(b_{U_{\lambda}}\right)$ is a subnet of (bU) in the compact set B, and hence has a cluster point $b \varepsilon$ B. Now let UEU be arbitrary. Find symmetric U'EU such that $U^{\prime} \subset U$. Find $\lambda_{0}$ such that $U^{\prime}<U_{\lambda_{0}}$.

Since $a_{U_{\lambda}} \rightarrow a$, we can find $\lambda_{1}>\lambda_{0}$ such that $\lambda>\lambda_{1} \Rightarrow a_{U_{\lambda}} \varepsilon U^{\prime}[a]$. Since b is a cluster point of $\left(b_{U_{\lambda}}\right)$ we can find $\lambda_{2} \geq \lambda_{1}$ such that $b_{U_{\lambda_{2}}} \varepsilon U^{\prime}[b]$. Now we have $\mathrm{a}_{\lambda_{2}} \varepsilon \mathrm{U}^{\prime}[\mathrm{a}], \mathrm{b}_{\mathrm{U}_{\lambda_{2}}} \varepsilon \mathrm{U}^{\prime}[\mathrm{b}], \mathrm{U}_{\lambda_{2}} \geqq \mathrm{U}_{\lambda_{1}} \geqq \mathrm{U}_{\lambda_{0}} \geqq \mathrm{U}^{\prime}$. Now there exists a $\mathrm{U}_{\lambda_{2}}$-chain in $\mathrm{X}$ joining $a_{\lambda_{2}}$ to $b_{U_{2}}$. Since $U_{\lambda_{2}} \subset U^{\prime}$, this is also a $U^{\prime}$-chain, and since $\left(a, a_{U_{\lambda}}\right) \varepsilon U^{\prime}$ and $\left(b, b_{U_{2}}\right) \varepsilon U^{\prime}$, there is thus a $U^{\prime}$-chain joining a to $b$, and hence a $U$-chain joining a to b. By Corollary 3.5, $a$ and $b$ lie together in the same component of $X$, contradicting the fact that no component of $X$ intersects both $A$ and $B$. Thus a $U \varepsilon U$ as claimed above exists.

Let $X_{A}=C_{U}(A)$ and $X_{B}=X-X_{A}$. By Proposition $3.1 x_{A}$ is clopen and hence $X_{A}$ and $X_{B}$ are compact. Furthermore $A \subset X_{A}$ is clear, $B \subset X_{B}$ follows by the above claim, and $\mathrm{X}_{\mathrm{A}} \cup \mathrm{X}_{\mathrm{B}}$ is a separation of $\mathrm{X}$. 


\section{REFERENCES}

1. MROWKA, S.G. and PERVIN, W.J., On uniform connectedness, Proc. Amer. Math. Soc. 15. , (1964), 446-449.

2. BABOOLAL, D., On Some Uniform Connection Properties related to Local Connectedness, Quaestiones Mathematicae 7 (1984), 155-160.

3. WHYBURN, G.T., Analytic Topology, Amer. Math. Soc. Collog. Publications, 28 (1942).

4. KELLEY, J.L., General Topology, Springer-Verlag, New York, He1delberg, Berlin, (1955).

5. NAIMPALLY, S.A. and WARRAC, B.D., Proximity Spaces, Cambridge Tracts in math. and Math. Phys., Cambridge (1970).

6. SIEBER, J.L. and PERVIN, W.J., Connectedness in syntopogenous spaces, Proc. Ame r. Math. Soc. 15 (1964), 590-595. 


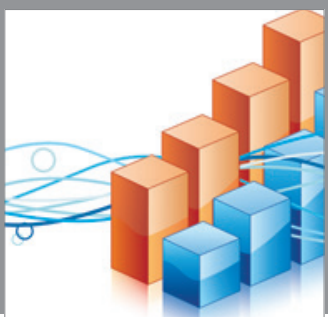

Advances in

Operations Research

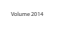

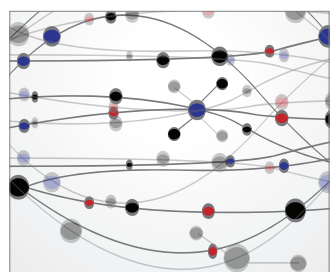

\section{The Scientific} World Journal
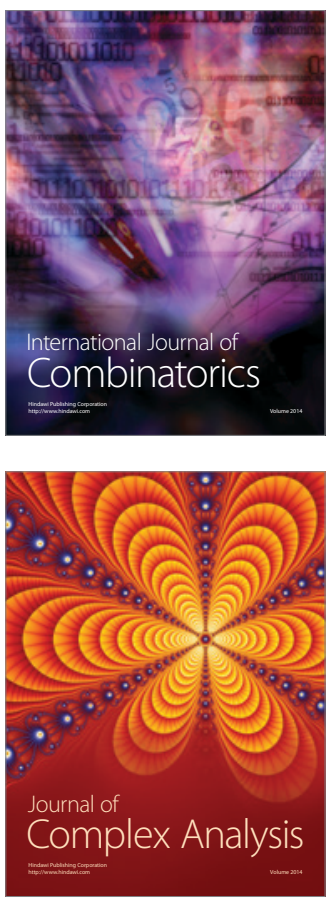

International Journal of

Mathematics and

Mathematical

Sciences
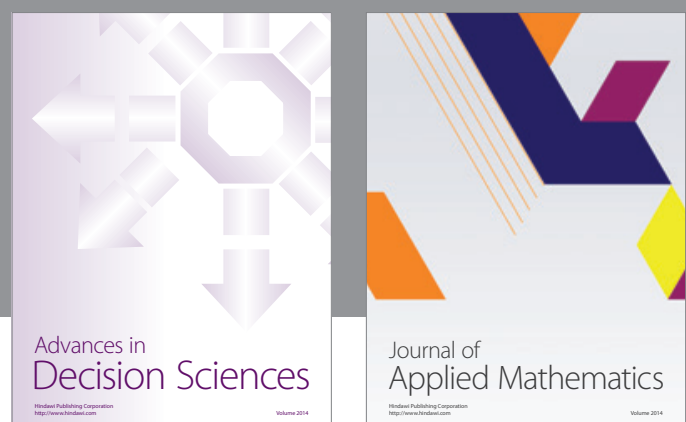

Journal of

Applied Mathematics
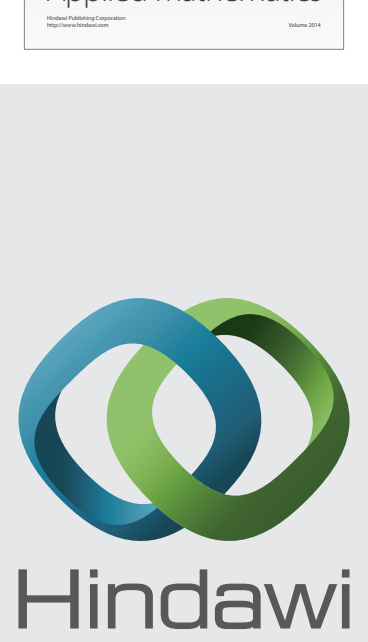

Submit your manuscripts at http://www.hindawi.com
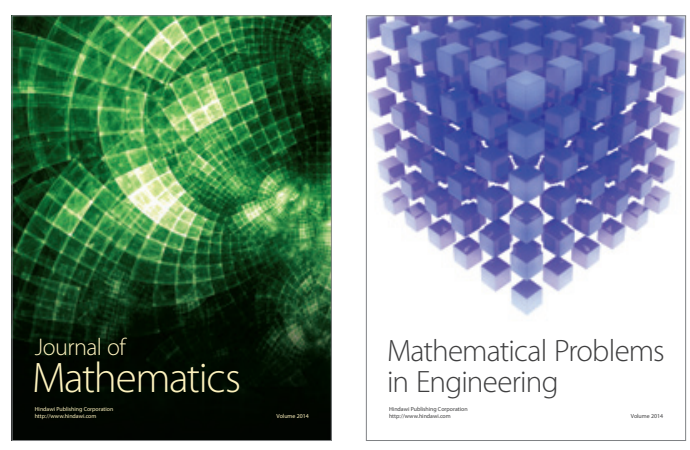

Mathematical Problems in Engineering
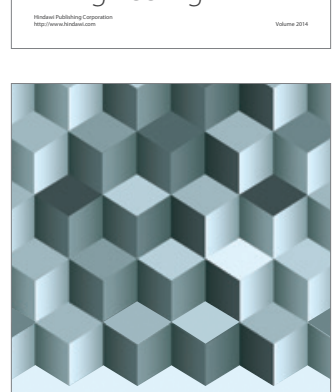

Journal of

Function Spaces
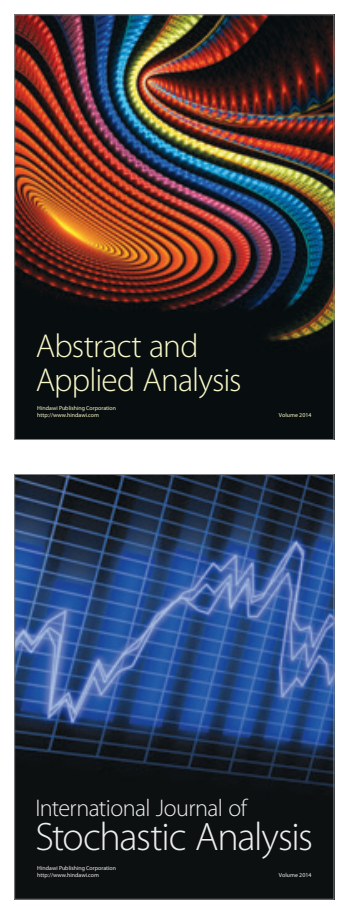

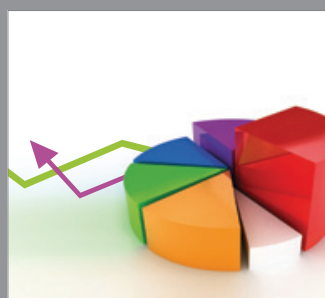

ournal of

Probability and Statistics

Promensencen
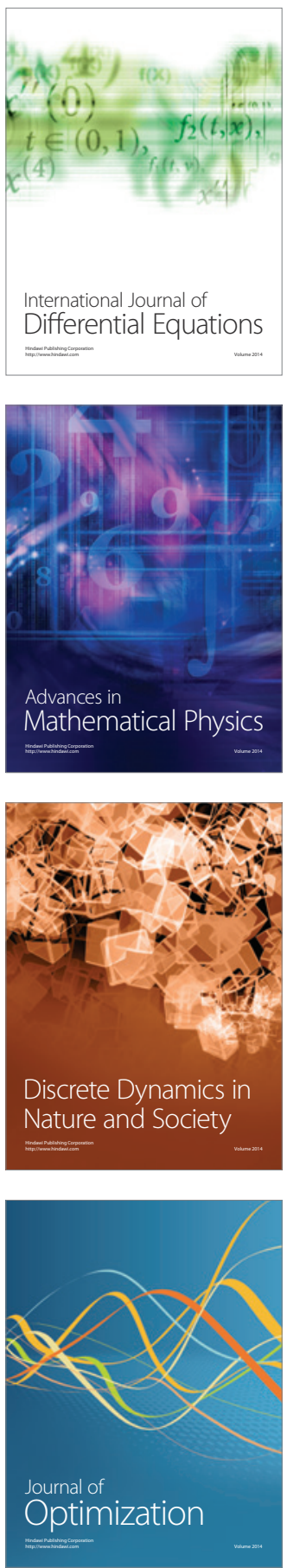